\title{
On compact realifications of exceptional simple Kantor triple systems
}

\author{
Daniel MONDOC \\ Department of Mathematics, Royal Institute of Technology (KTH), \\ Lindstedts väg 25, S-100 44 Stockholm, Sweden
}

E-mail:mondoc@math.kth.se

\begin{abstract}
Let $A$ be the realification of the matrix algebra determined by Jordan algebra of hermitian matrices of order three over a complex composition algebra. We define an involutive automorphism on $A$ with a certain action on the triple system obtained from $A$ which give models of simple compact Kantor triple systems. In addition, we give an explicit formula for the canonical trace form and the classification for these triples and their corresponding exceptional real simple Lie algebras. Moreover, we present all realifications of complex exceptional simple Lie algebras as Kantor algebras for a compact simple Kantor triple system defined on a structurable algebra of skew-dimension one.
\end{abstract}

2000 MSC: 17A40, 17A30, 17A75, 17B70, 17C20

\section{Introduction}

Models of Kantor triple systems defined on the $2 \times 2$-matrix algebra determined by the Jordan algebra $J=H_{3}\left(\mathbb{A}^{\mathbb{C}}\right)$ of hermitian $3 \times 3$-matrices over complex composition algebras $\mathbb{A}^{\mathbb{C}}$ considered over the field $\mathbb{C}$ of complex numbers appeared in a unified formula given by I. L. Kantor $[17,18]$ in connection with exceptional Lie algebras and a classification theorem over $\mathbb{C}$.

The notion of (simple) structurable algebras was given by B. N. Allison [1] who studied in particular those of skew-dimension one [3]. Moreover, the connection between Kantor triple systems and structurable algebras was studied by H. Asano and S. Kaneyuki [7] who also defined and studied $[5,15]$ compact Kantor triple systems in connection with classical real Lie algebras and a classification theorem over the field $\mathbb{R}$.

In this paper we continue the work on compact simple Kantor triple systems of [5] and $[20,21,22]$ giving, by a unified formula (Theorem 1), the classification of exceptional compact simple Kantor triple systems defined on the realification of the $2 \times 2$-matrix algebra determined by Jordan algebra $J=H_{3}\left(\mathbb{A}^{\mathbb{C}}\right)$ of hermitian $3 \times 3$-matrices over a complex composition algebra $\mathbb{A}^{\mathbb{C}}$ corresponding to realifications of complex exceptional simple Lie algebras (Theorem 2). In addition, we give an explicit formula for the quadratic canonical trace form for these Kantor triple systems (Corollary 1). Further, we present all realifications of complex exceptional simple Lie algebras as Kantor algebras for a compact simple Kantor triple system defined on a structurable algebra of skew-dimension one (Theorem 2, Proposition 5).

The results presented here are a continuation of [20] where models of exceptional compact simple Kantor triple systems defined on the $2 \times 2$-matrix algebra determined by Jordan algebra $J=H_{3}(\mathbb{A})$ of hermitian $3 \times 3$-matrices over a real composition algebra $\mathbb{A}$ have been given. Related results are those of [10] where a construction of exceptional simple 5-graded Lie algebras $\mathcal{U}=\oplus_{l=-2}^{2} U_{l}$ and an explicit realization of the subspaces $U_{l}$ have been given by different methods. Moreover, the notion of Kantor triple systems and their structure theory have been generalized by $(\epsilon, \delta)$-Freudenthal-Kantor triple systems [13, 24] such that Kantor triple systems coincide with $(-1,1)$-Freudenthal-Kantor triple systems. A realization of exceptional simple 5 -graded Lie algebras in terms of Freudenthal-Kantor triple systems have been given by N. Kamiya [14]. 
The models of compact simple Kantor triple systems considered here start with a structurable algebra $A$, its associated Kantor triple system $B_{A}(x, y, z):=V_{x, y}(z), x, y, z \in A$, and an involutive automorphism $\varphi$. Then the new triple product $B_{A}(x, \varphi(y), z)$ is considered, which gives again a Kantor triple system. Suitable elections of $A$ and $\varphi$ (here $\varphi(y)=\bar{y}^{\sim}$, where ${ }^{-}$is the standard involution and $\sim$ denotes a certain involution on $A$ ) give compact simple models.

The structure of this paper is as follows. Section 2 serves a preliminary purpose; we give a short overview of the basic definitions and known results on triple systems, graded Lie algebras and structurable algebras. The main results mentioned above, on $B_{A}(x, \varphi(y), z)$, the corresponding canonical trace form and the corresponding exceptional real simple Lie algebras are proved in section 3 .

\section{Triple systems, graded Lie algebras and structurable algebras}

Let $\mathcal{U}$ be a Lie algebra over a field $\mathbf{F}$ of characteristic zero. $\mathcal{U}$ is called a graded Lie algebra (abbreviated as GLA) if it is a Lie algebra of the form $\mathcal{U}=\oplus_{l=-\infty}^{\infty} U_{l}$ such that $\left[U_{l}, U_{k}\right] \subseteq U_{l+k}$.

A GLA $\mathcal{U}=\oplus_{l=-\infty}^{\infty} U_{l}$ is called 5-graded if $U_{ \pm n}=0$ for any integer $n>2$.

Let $U$ be a finite dimensional vector space over the field $\mathbf{F}$ and $B: U \times U \times U \rightarrow U$ be a trilinear map. The pair $(B, U)$ is called a triple system over $\mathbf{F}$.

For $x, y \in U$ define the linear endomorphisms $L_{x, y}, R_{x, y}$ and $S_{x, y}$ on $U$ by

$$
\begin{aligned}
& L_{x, y}(z):=B(x, y, z), \quad R_{x, y}(z):=B(z, x, y) \\
& S_{x, y}(z):=B(x, z, y)-B(y, z, x), \quad z \in U
\end{aligned}
$$

A triple system $(B, U)$ is called a generalized Jordan triple system (abbreviated as GJTS) if the following identity is valid [7] ( $\S 1)$ :

$$
\left[L_{x, y}, L_{u, v}\right]=L_{L_{x, y}(u), v}-L_{u, L_{y, x}(v)}, \quad u, v, x, y \in U
$$

Let $(B, U)$ and $\left(B^{\prime}, U^{\prime}\right)$ be two GJTS's. We say that a linear map $F$ of $U$ into $U^{\prime}$ is a homomorphism if $F$ satisfies the identity $F(B(x, y, z))=B^{\prime}(F(x), F(y), F(z))$, for all $x, y, z \in U$. Moreover, if $F$ is bijective, then $F$ is called an isomorphism. In this case the GJTS's $(B, U)$ and $\left(B^{\prime}, U^{\prime}\right)$ are said to be isomorphic.

Let $(B, U)$ be a GJTS and $V_{k}, k=1,2,3$, be subspaces of $U$. We denote by $B\left(V_{1}, V_{2}, V_{3}\right)$ the subspace of $U$ spanned by elements $B\left(x_{1}, x_{2}, x_{3}\right), x_{k} \in V_{k}, k=1,2,3$. A subspace $V$ of $U$ is called an ideal of $(B, U)$ if the following relations hold $B(V, U, U) \subseteq V, B(U, V, U) \subseteq V, B(U, U, V) \subseteq V$. The GJTS $(B, U)$ is called simple if $B$ is not a zero map and $(B, U)$ has no non-trivial ideal.

Starting from a given GJTS $(B, U)$, I. L. Kantor [17] constructed a certain GLA $\mathcal{L}(B)=$ $\oplus_{l=-\infty}^{\infty} U_{l}$ such that $U_{-1}=U$. The Lie algebra $\mathcal{L}(B)$ is called the Kantor algebra for $(B, U)[5]$. A GJTS $(B, U)$ is called of the $n$-th order if its Kantor algebra is of the form $\mathcal{L}(B)=\oplus_{l=-n}^{n} U_{l}$. We shall call a GJTS of the second order for short a Kantor triple system [4] (abbreviated as KTS). By [17] Proposition 10, a GJTS $(B, U)$ is a KTS if and only if

$$
S_{S_{x, y}(u), v}=S_{x, y} L_{u, v}+L_{v, u} S_{x, y}, \quad u, v, x, y \in U
$$

Remark. Many authors $[4,18]$ define a KTS to be a triple system $(B, U)$ satisfying the identities $(2.2),(2.3)$ instead of the identity (2.2) together with the fact that its Kantor algebra is 5-graded. The definitions are equivalent.

A GJTS is called exceptional (classical) if its Kantor algebra is exceptional (classical) Lie algebra.

For $z \in U$ we define a bilinear map $B_{z}$ on $U$ by $B_{z}(x, y)=B(x, z, y), x, y \in U$. We say that $(B, U)$ satisfies the condition (A) if $B_{z}=0$ implies $z=0$. 
Let $(B, U)$ be a finite dimensional KTS. We consider the symmetric bilinear form on $U[5]$

$$
\gamma_{B}(x, y):=\frac{1}{2} \operatorname{Tr}\left(2 R_{x, y}+2 R_{y, x}-L_{x, y}-L_{y, x}\right)
$$

where $\operatorname{Tr}(f)$ means the trace of a linear endomorphism $f$. We shall call the form $\gamma_{B}$ defined by (2.4) the canonical (trace) form for the $\operatorname{KTS}(B, U)$.

A finite dimensional KTS $(B, U)$ is called compact if its canonical form $\gamma_{B}$ is positive definite.

Let $A$ be an algebra over $\mathbf{F}$. Let left (right) multiplication $L_{x}: A \mapsto A,\left(R_{x}: A \mapsto A\right)$ be defined by $L_{x}(y)=x y,\left(R_{x}(y)=y x\right), x, y \in A$ and denote by $H_{o m}(A)$ the associative algebra over $\mathbf{F}$ of all linear transformations on $A$. If $A$ is finite $\operatorname{dimensional~we~} \operatorname{denote} \operatorname{by} \operatorname{dim}_{\mathbf{F}} A$ the dimension of $A$ over $\mathbf{F}$. For any extension field $K$ of $\mathbf{F}$ we denote $A^{K}=K \otimes_{\mathbf{F}} A$.

Proposition 1 ([11]). Let $A$ be a finite dimensional algebra over an algebraically closed field $\Gamma$ and let $\Phi$ be a subfield of $\Gamma$. If $A$ is simple over $\Gamma$ then $A$ is simple as algebra considered over $\Phi$.

Remark. A direct proof of Proposition 1 is available in [22].

Let $\left(A,{ }^{-}\right)$be a unital non-associative algebra over $\mathbf{F}$ with involution (involutive anti-automorphism $)^{-}$. We define $V_{x, y} \in \operatorname{Hom}_{\mathbf{F}}(A)$ and the triple system $B_{A}(x, y, z)$ by

$$
\begin{aligned}
& V_{x, y}:=L_{L_{x}(\bar{y})}+R_{x} R_{\bar{y}}-R_{y} R_{\bar{x}}, \quad x, y \in A \\
& B_{A}(x, y, z):=V_{x, y}(z)=(x \bar{y}) z+(z \bar{y}) x-(z \bar{x}) y, \quad x, y, z \in A
\end{aligned}
$$

$B_{A}(x, y, z)$ is called the triple system obtained from the algebra $\left(A,^{-}\right)[7](\S 2)$. We shall write for short $B_{A}$ for $\left(B_{A}, A\right)$.

A unital non-associative algebra with involution $\left(A,,^{-}\right)$is called a structurable algebra if the following identity is fulfilled [3]:

$$
\left[V_{x, y}, V_{u, v}\right]=V_{V_{x, y}(u), v}-V_{u, V_{y, x}(v)}, \quad u, v, x, y \in A
$$

Let $\left(A,^{-}\right)$be a structurable algebra. Then, by [3], $A=\mathcal{S} \oplus \mathcal{H}$, where

$$
\mathcal{S}:=\mathcal{S}\left(A,^{-}\right):=\{s \in A \mid \bar{s}=-s\} \quad \text { and } \quad \mathcal{H}:=\mathcal{H}\left(A,,^{-}\right):=\{h \in A \mid \bar{h}=h\}
$$

are the spaces of skew-hermitian and hermitian elements of $A$, respectively and $\operatorname{dim} \mathcal{S}$ is called the skew-dimension of $\left(A,^{-}\right)$.

To a structurable algebra $\left(A,{ }^{-}\right)$Allison [2] associated a 5-GLA $\mathcal{K}(A)$ as follows

$$
\begin{aligned}
& \mathcal{K}(A)=\oplus_{l=-2}^{2} K_{l}, \quad \text { where } \\
& K_{-2}=\mathcal{S}, \quad K_{-1}=A, \quad K_{0}=\left\{V_{x, y} \in \operatorname{Hom}_{\mathbf{F}}(A): x, y \in A\right\} \\
& K_{l}(l=1,2) \text { is an isomorphic copy of } K_{-l}
\end{aligned}
$$

By [7] Theorem 2.5, Allison's 5-GLA $\mathcal{K}(A)$ coincides with Kantor's 5-GLA $\mathcal{L}\left(B_{A}\right)$, where $B_{A}$ is the triple system obtained from the algebra $\left(A,^{-}\right)$.

Let $J$ be a finite dimensional separable degree 3 Jordan algebra over $\mathbf{F}$. Let $N, T$ and ${ }^{\sharp}$ be the norm form, trace form and adjoint map on $J$ respectively [12] (§6.3). Define $\times: J \times J \rightarrow J$ by $x \times y=(x+y)^{\sharp}-x^{\sharp}-y^{\sharp}$. Then the algebra $\mathcal{M}(J)$ with multiplication and standard involution - defined [3] (§1) by

$$
\begin{aligned}
& \mathcal{M}(J):=\left\{\left(\begin{array}{ll}
\xi_{1} & x_{1} \\
x_{2} & \xi_{2}
\end{array}\right) \mid \xi_{1}, \xi_{2} \in \mathbf{F}, x_{1}, x_{2} \in J\right\} \\
& \left(\begin{array}{ll}
\xi_{1} & x_{1} \\
x_{2} & \xi_{2}
\end{array}\right)\left(\begin{array}{ll}
\eta_{1} & y_{1} \\
y_{2} & \eta_{2}
\end{array}\right):=\left(\begin{array}{cc}
\xi_{1} \eta_{1}+T\left(x_{1}, y_{2}\right) & \xi_{1} y_{1}+\eta_{2} x_{1}+x_{2} \times y_{2} \\
\eta_{1} x_{2}+\xi_{2} y_{2}+x_{1} \times y_{1} & \xi_{2} \eta_{2}+T\left(x_{2}, y_{1}\right)
\end{array}\right)
\end{aligned}
$$




$$
\overline{\left(\begin{array}{ll}
\xi_{1} & x_{1} \\
x_{2} & \xi_{2}
\end{array}\right)}:=\left(\begin{array}{ll}
\xi_{2} & x_{1} \\
x_{2} & \xi_{1}
\end{array}\right)
$$

is called the $2 \times 2$-matrix algebra determined by Jordan algebra $J$.

Let $\mathbb{R}, \mathbb{C}, \mathbb{H}$ and $\mathbb{O}$ denote the real algebras of real and complex numbers, quaternions and octonions, respectively. They are called division composition algebras and are defined by their explicit multiplication tables [23].

Let $\mathbb{A}$ be any of the division composition algebras $\mathbb{R}, \mathbb{C}, \mathbb{H}, \mathbb{O}$ and let $u_{l}, l=1, \ldots, \operatorname{dim}_{\mathbb{R}} \mathbb{A}$, where $\operatorname{dim}_{\mathbb{R}} \mathbb{A} \in\{1,2,4,8\}$, denote the standard units of $\mathbb{A}$. We define conjugation - on a standard unit $u$ of $\mathbb{A}$ by

$$
\overline{1}=1, \quad \bar{u}=-u, \quad \text { if } \quad u \neq 1
$$

and extend conjugation - by linearity on $\mathbb{A}$.

Remark. By [23] $\S 3$, it is known that ${ }^{-}$is an involution on any composition algebra $\mathbb{A}$ above.

Let now $\mathbb{A}^{\mathbb{C}}$ be any of the complex composition algebras $\mathbb{R}^{\mathbb{C}}, \mathbb{C}^{\mathbb{C}}, \mathbb{H}^{\mathbb{C}}, \mathbb{O}^{\mathbb{C}}$, i.e. the division composition algebras regarded as algebras over $\mathbb{C}$. We define conjugation ${ }^{-}$and scalar extended conjugation $^{\wedge}$, called pseudoconjugation, on the complex algebra $\mathbb{A}^{\mathbb{C}}$ by

$$
\begin{aligned}
& \bar{x}=\left(\sum_{l=1}^{\operatorname{dim}_{\mathbb{C}} \mathbb{A}^{\mathbb{C}}} \alpha_{l} u_{l}\right)^{-}:=\sum_{l=1}^{\operatorname{dim}_{\mathbb{C}} \mathbb{A}^{\mathbb{C}}} \alpha_{l} \overline{u_{l}}, \alpha_{l} \in \mathbb{C} \\
& x^{\wedge}=\left(\sum_{l=1}^{\operatorname{dim}_{\mathbb{C}} \mathbb{A}^{\mathbb{C}}} \alpha_{l} u_{l}\right)^{\wedge}:=\sum_{l=1}^{\operatorname{dim}_{\mathbb{C}} \mathbb{A}^{\mathbb{C}}} \overline{\alpha_{l}} \overline{u_{l}}, \alpha_{l} \in \mathbb{C}
\end{aligned}
$$

where $x=\sum_{l=1}^{\operatorname{dim}_{\mathbb{C}} \mathbb{A}^{\mathbb{C}}} \alpha_{l} u_{l}$ is an arbitrary element of $\mathbb{A}^{\mathbb{C}}, u_{l}$ are the standard units and $\overline{u_{l}}$ is defined by (2.8) hence $\overline{\alpha_{l}}$ is the standard complex conjugate of $\alpha_{l}$.

Remark. Then clearly ${ }^{-}$and ${ }^{\wedge}$ are involutions of the complex algebra $\mathbb{A}^{\mathbb{C}}$.

Let $\mathbb{A}$ be each one of the division composition algebras $\mathbb{R}, \mathbb{C}, \mathbb{H}, \mathbb{O}$.

Let $\mathcal{A} \in\left\{\mathbb{A}, \mathbb{A}^{\mathbb{C}}\right\}$ denote any of the real or complex composition algebras and let $\mathcal{M}_{3}(\mathcal{A})$ denote the set of matrices of order 3 with entries in $\mathcal{A}$. Then, by definition, the conjugation on the algebra $\mathcal{A}=\mathbb{A}$ and the conjugation ${ }^{-}$and pseudoconjugation ${ }^{\wedge}$ on the algebra $\mathcal{A}=\mathbb{A}^{\mathbb{C}}$ are induced on $\mathcal{M}_{3}(\mathcal{A})$ by ${ }^{-}$and ${ }^{\wedge}$, respectively, on each entry.

Remark. Clearly ${ }^{-}$and ${ }^{\wedge}$, respectively, are involutive on $\mathcal{M}_{3}(\mathcal{A})$.

Let $H_{3}(\mathcal{A}):=\left\{x \in \mathcal{M}_{3}(\mathcal{A}) \mid \bar{x}^{T}=x\right\}$ denote the Jordan algebra of hermitian $3 \times 3$-matrices over a composition algebra $\mathcal{A} \in\left\{\mathbb{A}, \mathbb{A}^{\mathbb{C}}\right\}[8](\S 6)$ with the product

$$
x \cdot y=\frac{1}{2}(x y+y x), \quad x, y \in H_{3}(\mathcal{A})
$$

where in the right hand side we have usual matrix multiplication and $\bar{x}^{T}$ denotes the conjugate transposed of $x \in \mathcal{M}_{3}(\mathcal{A})$. Then, by [9] (p. 218), the trace form and $\times$-operation in formulas (2.7) are defined on $H_{3}(\mathcal{A})$ by

$$
\begin{aligned}
& T(x, y)=\operatorname{Tr}(x \cdot y)=\frac{1}{2} \operatorname{Tr}(x y+y x) \\
& x \times y=x \cdot y-\frac{1}{2}[\operatorname{Tr}(x) y+\operatorname{Tr}(y) x]+\frac{1}{2}[\operatorname{Tr}(x) \operatorname{Tr}(y)-\operatorname{Tr}(x \cdot y)] I_{3}
\end{aligned}
$$

for all $x, y \in H_{3}(\mathcal{A})$, where $I_{3}$ is the unit matrix of order 3 and $\operatorname{Tr}(x)$ denotes the trace of $x$. 
Lemma $1([3])$. Let $\left(A,^{-}\right):=\mathcal{M}\left(H_{3}\left(\mathbb{A}^{\mathbb{C}}\right)\right)$ be the $2 \times 2$-matrix algebra determined by the Jordan algebra $H_{3}\left(\mathbb{A}^{\mathbb{C}}\right)$ of hermitian $3 \times 3$-matrices over a complex composition algebra $\mathbb{A}^{\mathbb{C}} \in$ $\left\{\mathbb{R}^{\mathbb{C}}, \mathbb{C}^{\mathbb{C}}, \mathbb{H}^{\mathbb{C}}, \mathbb{O}^{\mathbb{C}}\right\}$, where $\left(^{-}\right)$is the standard involution on $A$. Then, over $\mathbb{C}$, the algebras $\left(A,^{-}\right)$ are simple structurable of skew-dimension 1.

Proof. The assertion follows directly from [3] (§1 Proposition 1.10).

Let now $A$ be a K-algebra for any extension field of $K$ of $\mathbf{F}$. We denote by $A_{\mathbf{F}}$ the algebra $A$ considered as algebra over $\mathbf{F}$. If $A$ is an algebra over $\mathbb{C}$ then we call the algebra $A_{\mathbb{R}}$ the realification of the complex algebra $A$. Further, if $B_{A}$ is the triple system obtained from a complex algebra $\left(A,^{-}\right)$then we call the triple system $B_{A_{\mathbb{R}}}$ obtained from algebra $\left(A_{\mathbb{R}},^{-}\right)$the realification of $B_{A}$.

Proposition 2 ([22] Proposition 1.4). Let $\left(A,^{-}\right)$be a structurable algebra over $\mathbb{C}$. Then, over $\mathbb{R}$, the triple system $B_{A_{\mathbb{R}}}$ is a KTS satisfying the condition $(A)$, and $B_{A_{\mathbb{R}}}$ is simple if and only if $\left(A_{\mathbb{R}},{ }^{-}\right)$is simple.

\section{On compact realifications of exceptional simple Kantor triple systems}

We show first a property of the trace form on the Jordan algebra $\left(H_{3}\left(\mathbb{A}^{\mathbb{C}}\right),{ }^{-},{ }^{\wedge}\right)$.

Let $\epsilon_{l m}$ denote in $\mathcal{M}_{3}(\mathbb{R})$ the square matrix with entry 1 where the $l$-th row and the $m$-th column meet, all other entries being 0 , and denote

$$
\begin{array}{lll}
e_{1}=\epsilon_{11}, & e_{2}=\epsilon_{22}, & e_{3}=\epsilon_{33} \\
f_{1}=\epsilon_{12}+\epsilon_{21}, & f_{2}=\epsilon_{13}+\epsilon_{31}, & f_{3}=\epsilon_{23}+\epsilon_{32} \\
g_{1}=\epsilon_{12}-\epsilon_{21}, & g_{2}=\epsilon_{13}-\epsilon_{31}, & g_{3}=\epsilon_{23}-\epsilon_{32}
\end{array}
$$

Let $\mathbb{A}^{\mathbb{C}}$ denote any of the complex composition algebras $\mathbb{R}^{\mathbb{C}}, \mathbb{C}^{\mathbb{C}}, \mathbb{H}^{\mathbb{C}}, \mathbb{O}^{\mathbb{C}}$. From now on an arbitrary element of the Jordan algebra $H_{3}\left(\mathbb{A}^{\mathbb{C}}\right)$ is of the form

$$
x=\left(\begin{array}{lll}
x_{11} & x_{12} & x_{13} \\
\overline{x_{12}} & x_{22} & x_{23} \\
\overline{x_{13}} & \overline{x_{23}} & x_{33}
\end{array}\right), \quad x_{l l} \in \mathbb{C}, x_{l m} \in \mathbb{A}^{\mathbb{C}}, 1 \leq l<m \leq 3
$$

Lemma 2. Let $x$ be an arbitrary element in $H_{3}\left(\mathbb{A}^{\mathbb{C}}\right)$ of the form (3.2) and let the trace form $T(x, y), x, y \in H_{3}\left(\mathbb{A}^{\mathbb{C}}\right)$ be defined by (2.11). Let ${ }^{-}$and ${ }^{\wedge}$ be the conjugation and pseudoconjugation defined on $H_{3}\left(\mathbb{A}^{\mathbb{C}}\right)$ by (2.9) and (2.10), respectively. Then

$$
T\left(x, \bar{x}^{\wedge}\right)=\sum_{1 \leq l, m \leq 3}\left\|x_{l m}\right\|^{2}
$$

where $\left\|x_{l m}\right\|$ denotes the norm of $x_{l m}, 1 \leq l, m \leq 3$.

Proof. Let $x=\left(x_{l m}\right)$ be an arbitrary element in $H_{3}\left(\mathbb{A}^{\mathbb{C}}\right)$ of the form $(3.2)$, where $x_{l m} \in$ $\mathbb{A}^{\mathbb{C}}, 1 \leq l, m \leq 3$. Let $x_{l m}=c_{l m} \otimes_{\mathbb{R}} a_{l m}, c_{l m} \in \mathbb{C}, a_{l m} \in \mathbb{A}$. We write for short $\otimes_{\mathbb{R}}=\otimes$. Then $\overline{x_{l m}}=c_{l m} \otimes \overline{a_{l m}}$, by $(2.9)$, and $x_{l m}^{\wedge}=\overline{c_{l m}} \otimes \overline{a_{l m}}$, by (2.10), where ${ }^{-}$is defined by (2.8) so $\overline{c_{l m}}$ is the standard complex conjugate of $c_{l m}$. Hence, $\overline{x_{l m}} \wedge=\overline{c_{l m}} \otimes a_{l m}$ and by (3.2) we have

$$
x=\left(\begin{array}{ccc}
c_{11} & c_{12} \otimes a_{12} & c_{13} \otimes a_{13} \\
c_{12} \otimes \overline{a_{12}} & c_{22} & c_{23} \otimes a_{23} \\
c_{13} \otimes \overline{a_{13}} & c_{23} \otimes \overline{a_{23}} & c_{33}
\end{array}\right)
$$


and

$$
\bar{x}^{\wedge}=\left(\begin{array}{ccc}
\overline{c_{11}} & \overline{c_{12}} \otimes a_{12} & \overline{c_{13}} \otimes a_{13} \\
\overline{c_{12}} \otimes \overline{a_{12}} & \overline{c_{22}} & \overline{c_{23}} \otimes a_{23} \\
\overline{c_{13}} \otimes \overline{a_{13}} & \overline{c_{23}} \otimes \overline{a_{23}} & \overline{c_{33}}
\end{array}\right)
$$

Then, by (2.11), (3.3) and (3.4), straightforward calculations give

$$
T\left(x, \bar{x}^{\wedge}\right)=\sum_{1 \leq l \leq 3}\left\|c_{l l}\right\|^{2}+2 \sum_{1 \leq l<m \leq 3}\left\|c_{l m}\right\|^{2}\left\|a_{l m}\right\|^{2}=\sum_{1 \leq l, m \leq 3}\left\|x_{l m}\right\|^{2}
$$

where $\left\|x_{l m}\right\|$ denotes the norm of $x_{l m}, 1 \leq l, m \leq 3$.

\subsection{The exceptional simple Lie algebras $F_{4 \mathbb{R}}^{\mathbb{C}}, E_{6 \mathbb{R}}^{\mathbb{C}}, E_{7 \mathbb{R}}^{\mathbb{C}}$ and $E_{8 \mathbb{R}}^{\mathbb{C}}$}

We define now models of compact simple Kantor triple systems.

Let $\mathbb{A}^{\mathbb{C}}$ denote any of the complex composition algebras $\mathbb{R}^{\mathbb{C}}, \mathbb{C}^{\mathbb{C}}, \mathbb{H}^{\mathbb{C}}, \mathbb{O}^{\mathbb{C}}$.

Let $\mathcal{M}\left(H_{3}\left(\mathbb{A}^{\mathbb{C}}\right)\right)$ be the $2 \times 2$-matrix algebra determined by the Jordan algebra $H_{3}\left(\mathbb{A}^{\mathbb{C}}\right)$ defined by (2.7) with standard involution ${ }^{-}$. Let us define a second involution $\sim$ on $\mathcal{M}\left(H_{3}\left(\mathbb{A}^{\mathbb{C}}\right)\right)$ by

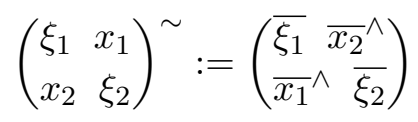

where $\overline{x_{i}} \wedge$ denotes the pseudo-conjugate of $x_{i}$ conjugate, $x_{i} \in H_{3}\left(\mathbb{A}^{\mathbb{C}}\right)$ and $\overline{\xi_{i}}$ is the standard conjugate of $\xi_{i} \in \mathbb{C}, i=1,2$. Hence the following involutive automorphism is defined on $\mathcal{M}\left(H_{3}\left(\mathbb{A}^{\mathbb{C}}\right)\right)$

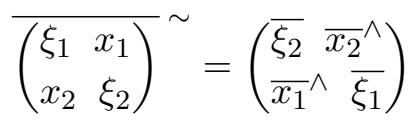

Let $\mathcal{M}\left(H_{3}\left(\mathbb{A}^{\mathbb{C}}\right)\right)_{\mathbb{R}}$ be the realification of the algebra $\mathcal{M}\left(H_{3}\left(\mathbb{A}^{\mathbb{C}}\right)\right)$ and let $\left(\phi, \mathcal{M}\left(H_{3}\left(\mathbb{A}^{\mathbb{C}}\right)\right)_{\mathbb{R}}\right)$ denote the triple system defined by formula

$$
\phi(x, y, z)=\left(x y^{\sim}\right) z+\left(z y^{\sim}\right) x-(z \bar{x}) \bar{y}^{\sim}, \quad x, y, z \in \mathcal{M}\left(H_{3}\left(\mathbb{A}^{\mathbb{C}}\right)\right)_{\mathbb{R}}
$$

We prove now the main results in the Theorems 1, 2 and Corollary 1.

Proposition 3. The triple systems $\left(\phi, \mathcal{M}\left(H_{3}\left(\mathbb{A}^{\mathbb{C}}\right)\right)_{\mathbb{R}}\right)$ defined by (3.5), (3.7) are KTS's satisfying the condition (A).

Proof. From Lemma 1 and Propositions 1, 2 follows that the triple systems $B_{\mathcal{M}\left(H_{3}\left(\mathbb{A}^{\mathbb{C}}\right)\right)_{\mathbb{R}}}(x, y, z)$, $x, y, z \in \mathcal{M}\left(H_{3}\left(\mathbb{A}^{\mathbb{C}}\right)\right)_{\mathbb{R}}$ are (simple) KTS's satisfying the condition (A). Further, we remark that $\phi(x, y, z)=B_{\mathcal{M}\left(H_{3}\left(\mathbb{A}^{\mathbb{C}}\right)\right)_{\mathbb{R}}}\left(x, \bar{y}^{\sim}, z\right)$, by (3.7) and (2.5). Then the assertions follow from [6] Lemma 1.5 since the map $\varphi(y)=\bar{y}^{\sim}$ is an involutive automorphism on the algebra $\left.\mathcal{M}\left(H_{3}\left(\mathbb{A}^{\mathbb{C}}\right)\right)_{\mathbb{R}}\right)$.

Theorem 1. The KTS's $\left(\phi, \mathcal{M}\left(H_{3}\left(\mathbb{A}^{\mathbb{C}}\right)\right)_{\mathbb{R}}\right)$ defined by (3.5), (3.7) on the realification of the $2 \times 2$-matrix algebra determined by Jordan algebra $H_{3}\left(\mathbb{A}^{\mathbb{C}}\right)$ of hermitian $3 \times 3$-matrices over $\mathbb{A}^{\mathbb{C}} \in\left\{\mathbb{R}^{\mathbb{C}}, \mathbb{C}^{\mathbb{C}}, \mathbb{H}^{\mathbb{C}}, \mathbb{O}^{\mathbb{C}}\right\}$ are compact and simple.

Proof. We prove first compactness. We must show that the canonical (trace) form $\gamma_{\phi}$ defined by (2.4) for the KTS's $\left(\phi, \mathcal{M}\left(H_{3}\left(\mathbb{A}^{\mathbb{C}}\right)\right)_{\mathbb{R}}\right)$ is positive definite. Since the canonical form is symmetric let us consider the corresponding quadratic form which, by (2.4), is equal to

$$
\gamma_{\phi}(x, x)=\operatorname{Tr}(f(x, x)), \quad \text { where } \quad f(x, x)=2 R_{x, x}-L_{x, x}, x \in \mathcal{M}\left(H_{3}\left(\mathbb{A}^{\mathbb{C}}\right)\right)_{\mathbb{R}}
$$


We remark first that

$$
f(x, x)=2 R_{x, x}-L_{x, x}=2\left(R_{x, x}-L_{x, x}\right)+L_{x, x}=2 g(x, x)+L_{x, x}
$$

where $g(x, x):=R_{x, x}-L_{x, x}$. Hence, by (3.8), we have

$$
\gamma_{\phi}(x, x)=\operatorname{Tr}(f(x, x))=2 \operatorname{Tr}(g(x, x))+\operatorname{Tr}\left(L_{x, x}\right)
$$

such that, by (3.7) and (2.1),

$$
\begin{aligned}
& L_{x, x}(z)=\left(x x^{\sim}\right) z+\left(z x^{\sim}\right) x-(z \bar{x}) \bar{x}^{\sim} \\
& g(x, x)(z)=(z \bar{x}-x \bar{z}) \bar{x}^{\sim}=(z \bar{x}-\bar{z} \bar{x}) \bar{x}^{\sim}
\end{aligned}
$$

where in the last equality we have used that ${ }^{-}$is an involution on $\mathcal{M}\left(H_{3}\left(\mathbb{A}^{\mathbb{C}}\right)\right)_{\mathbb{R}}$.

We calculate $\operatorname{Tr}(g(x, x))$ first. For this, we remark that for

$$
x:=\left(\begin{array}{ll}
\xi_{1} & x_{1} \\
x_{2} & \xi_{2}
\end{array}\right), \quad y:=\left(\begin{array}{ll}
\eta_{1} & y_{1} \\
y_{2} & \eta_{2}
\end{array}\right), \quad z:=\left(\begin{array}{ll}
\nu_{1} & z_{1} \\
z_{2} & \nu_{2}
\end{array}\right), \quad s_{0}:=\left(\begin{array}{lr}
1 & O_{3} \\
O_{3} & -1
\end{array}\right)
$$

$x, y, z, s_{0} \in \mathcal{M}\left(H_{3}\left(\mathbb{A}^{\mathbb{C}}\right)\right)_{\mathbb{R}}$, where $O_{3}$ denotes the zero matrix of order 3 , the identity $y-\bar{y}=$ $\left(\eta_{1}-\eta_{2}\right) s_{0}$ follows from (2.7). Hence

$$
z \bar{x}-\overline{z \bar{x}}=\left(\nu_{1} \xi_{2}-\nu_{2} \xi_{1}+T\left(z_{1}, x_{2}\right)-T\left(z_{2}, x_{1}\right)\right) s_{0}
$$

by (2.7), and then the identity

$$
g(x, x)(z)=\left(\nu_{1} \xi_{2}-\nu_{2} \xi_{1}+T\left(z_{1}, x_{2}\right)-T\left(z_{2}, x_{1}\right)\right) s_{0} \bar{x}^{\sim}
$$

follows from (3.11). Then, by (2.7), (3.5) and (3.12), we have

$$
g(x, x)(z)=\left(\nu_{1} \xi_{2}-\nu_{2} \xi_{1}+T\left(z_{1}, x_{2}\right)-T\left(z_{2}, x_{1}\right)\right)\left(\begin{array}{cc}
\overline{\xi_{2}} & \overline{x_{2}} \wedge \\
-\overline{x_{1}} \wedge & -\overline{\xi_{1}}
\end{array}\right)
$$

Recall that for any linear map $f: U \rightarrow \mathbf{F}, U$ a vector space over the field $\mathbf{F}$, yields $\operatorname{Tr}(f(\cdot) v)=$ $f(v)$ and let $f_{x}: \mathcal{M}\left(H_{3}\left(\mathbb{A}^{\mathbb{C}}\right)\right) \rightarrow \mathbb{C}$ be the linear map

$$
f_{x}:\left(\begin{array}{cc}
\nu_{1} & z_{1} \\
z_{2} & \nu_{2}
\end{array}\right) \mapsto \nu_{1} \xi_{2}-\nu_{2} \xi_{1}+T\left(z_{1}, x_{2}\right)-T\left(z_{2}, x_{1}\right)
$$

Then by (3.13) follows $g(x, x)=f_{x}(\cdot) v$ with $v=\left(\begin{array}{c}\overline{\xi_{2}} \\ -\overline{x_{1}} \wedge \\ -\overline{x_{2}} \wedge\end{array}\right)$, so

$$
\operatorname{Tr}(g(x, x))=2\left(\left\|\xi_{1}\right\|^{2}+\left\|\xi_{2}\right\|^{2}+T\left(x_{1},{\overline{x_{1}}}^{\wedge}\right)+T\left(x_{2},{\overline{x_{2}}}^{\wedge}\right)\right)
$$

for all $x=\left(\begin{array}{ll}\xi_{1} & x_{1} \\ x_{2} & \xi_{2}\end{array}\right) \in \mathcal{M}\left(H_{3}\left(\mathbb{A}^{\mathbb{C}}\right)\right)_{\mathbb{R}}$, where the factor 2 in (3.14) follows from the fact that the trace is calculated over the realification $\mathcal{M}\left(H_{3}\left(\mathbb{A}^{\mathbb{C}}\right)\right)_{\mathbb{R}}$.

We calculate now $\operatorname{Tr}\left(L_{x, x}\right)$. For this, we remark that by (3.10) and the fact that ${ }^{-}$and $\sim$ are involutive on $\mathcal{M}\left(H_{3}\left(\mathbb{A}^{\mathbb{C}}\right)\right)_{\mathbb{R}}$ follows

$$
L_{x, x}=L_{x x^{\sim}}+h_{x}-h_{\bar{x}^{\sim}}, \quad \text { where } \quad h_{x}(z):=\left(z x^{\sim}\right) x, \quad \forall x, z \in \mathcal{M}\left(H_{3}\left(\mathbb{A}^{\mathbb{C}}\right)\right)_{\mathbb{R}}
$$

Further, we calculate $\operatorname{Tr}\left(L_{x x^{\sim}}\right)$. By $(2.7),(3.5)$ and (3.12) we have

$$
x x^{\sim}=\left(\begin{array}{ll}
\xi_{1} & x_{1} \\
x_{2} & \xi_{2}
\end{array}\right)\left(\begin{array}{ll}
\overline{\xi_{1}} \overline{x_{2}} \wedge \\
\overline{x_{1}} \wedge \overline{\xi_{2}}
\end{array}\right)=\left(\begin{array}{ll}
\left\|\xi_{1}\right\|^{2}+T\left(x_{1}, \overline{x_{1}} \wedge\right) & \xi_{1} \overline{x_{2}} \wedge+\overline{\xi_{2}} x_{1}+\overline{x_{1}} \wedge \times x_{2} \\
\overline{\xi_{1}} x_{2}+\xi_{2} \overline{x_{1}} \wedge+x_{1} \times \overline{x_{2}} \wedge & \left\|\xi_{2}\right\|^{2}+T\left(x_{2}, \overline{x_{2}} \wedge\right)
\end{array}\right)
$$


Let us denote the units of $\mathcal{M}\left(H_{3}\left(\mathbb{A}^{\mathbb{C}}\right)\right)_{\mathbb{R}}$ by

$$
\begin{aligned}
& \mu_{1}=\left(\begin{array}{cc}
1 & O_{3} \\
O_{3} & 0
\end{array}\right), \quad \mu_{i 1}=\left(\begin{array}{cc}
i & O_{3} \\
O_{3} & 0
\end{array}\right), \quad \mu_{2}=\left(\begin{array}{cc}
0 & O_{3} \\
O_{3} & 1
\end{array}\right), \quad \mu_{i 2}=\left(\begin{array}{cc}
0 & O_{3} \\
O_{3} & i
\end{array}\right) \\
& \mu_{e_{l 3}}=\left(\begin{array}{cc}
0 & e_{l} \\
O_{3} & 0
\end{array}\right), \quad \mu_{i e_{l} 3}=\left(\begin{array}{cc}
0 & i e_{l} \\
O_{3} & 0
\end{array}\right), \quad \mu_{e_{l 4}}=\left(\begin{array}{cc}
0 & O_{3} \\
e_{l} & 0
\end{array}\right), \quad \mu_{i e_{l 4}}=\left(\begin{array}{cc}
0 & O_{3} \\
i e_{l} & 0
\end{array}\right) \\
& \mu_{f_{l 3}}=\left(\begin{array}{cc}
0 & f_{l} \\
O_{3} & 0
\end{array}\right), \quad \mu_{i f_{l 3}}=\left(\begin{array}{cc}
0 & i f_{l} \\
O_{3} & 0
\end{array}\right), \quad \mu_{f_{l 4}}=\left(\begin{array}{cc}
0 & O_{3} \\
f_{l} & 0
\end{array}\right), \quad \mu_{i f_{l 4}}=\left(\begin{array}{cc}
0 & O_{3} \\
i f_{l} & 0
\end{array}\right) \\
& \mu_{u_{n} g_{l 3}}=\left(\begin{array}{cc}
0 & u_{n} g_{l} \\
O_{3} & 0
\end{array}\right), \quad \mu_{i u_{n} g_{l 3}}=\left(\begin{array}{cc}
0 & i u_{n} g_{l} \\
O_{3} & 0
\end{array}\right) \\
& \mu_{u_{n} g_{l 4}}=\left(\begin{array}{cc}
0 & O_{3} \\
u_{n} g_{l} & 0
\end{array}\right), \quad \mu_{i u_{n} g_{l 4}}=\left(\begin{array}{cc}
0 & O_{3} \\
i u_{n} g_{l} & 0
\end{array}\right)
\end{aligned}
$$

where $e_{l}, i e_{l}, f_{l}, i f_{l}, u_{n} g_{l}, i u_{n} g_{l}\left(l=1,2,3, n=2, \ldots, \operatorname{dim}_{\mathbb{C}} \mathbb{A}^{\mathbb{C}}\right)$ is the basis of $H_{3}\left(\mathbb{A}^{\mathbb{C}}\right)_{\mathbb{R}}$ such that $e_{l}, f_{l}, g_{l}$ are defined by $(3.1), i$ denotes the complex unit and $u_{n} \neq 1$ are the standard units.

Let $c_{\mu}\left(L_{x x^{\sim}}(\mu)\right)$ denote the coefficient of a generic unit $\mu$ of $\mathcal{M}\left(H_{3}\left(\mathbb{A}^{\mathbb{C}}\right)\right)_{\mathbb{R}}$ in $L_{x x^{\sim}}(\mu)$. Then, by (3.16), straightforward calculations give

$$
\begin{aligned}
& c_{\mu_{1}}\left(L_{x x} \sim\left(\mu_{1}\right)\right)=c_{\mu_{i 1}}\left(L_{x x} \sim\left(\mu_{i 1}\right)\right)=c_{\mu_{3}}\left(L_{x x} \sim\left(\mu_{3}\right)\right)=\left\|\xi_{1}\right\|^{2}+T\left(x_{1}, \bar{x}_{1} \wedge\right) \\
& c_{\mu_{2}}\left(L_{x x} \sim\left(\mu_{2}\right)\right)=c_{\mu_{i 2}}\left(L_{x x^{\prime}} \sim\left(\mu_{i 2}\right)\right)=c_{\mu_{4}}\left(L_{x x^{\sim}}\left(\mu_{4}\right)\right)=\left\|\xi_{2}\right\|^{2}+T\left(x_{2},{\overline{x_{2}}}^{\wedge}\right)
\end{aligned}
$$

for all $\mu_{t} \in\left\{\mu_{e_{l t}}, \mu_{i e_{l} t}, \mu_{f_{l t}}, \mu_{i f_{l t}}, \mu_{u_{n} g_{l} t}, \mu_{i u_{n} g_{l t}}\right\}, t=3,4$. Then, by (3.17),

$$
\operatorname{Tr}\left(L_{x x^{\sim}}\right)=2\left(1+\operatorname{dim}_{\mathbb{C}} H_{3}\left(\mathbb{A}^{\mathbb{C}}\right)\right)\left[\left\|\xi_{1}\right\|^{2}+\left\|\xi_{2}\right\|^{2}+T\left(x_{1},{\overline{x_{1}}}^{\wedge}\right)+T\left(x_{2},{\overline{x_{2}}}^{\wedge}\right)\right]
$$

for all $x=\left(\begin{array}{ll}\xi_{1} & x_{1} \\ x_{2} & \xi_{2}\end{array}\right) \in \mathcal{M}\left(H_{3}\left(\mathbb{A}^{\mathbb{C}}\right)\right)_{\mathbb{R}}$.

We show now that $\operatorname{Tr}\left(h_{x}\right)=\operatorname{Tr}\left(h_{\bar{x}^{\sim}}\right)$, for all $x \in \mathcal{M}\left(H_{3}\left(\mathbb{A}^{\mathbb{C}}\right)\right)_{\mathbb{R}}$, where $h_{x}$ is defined by (3.15).

We remark first that $h_{x}(z):=\left(z x^{\sim}\right) x=(z \overline{\varphi(x)}) x, x, z \in \mathcal{M}\left(H_{3}\left(\mathbb{A}^{\mathbb{C}}\right)\right)_{\mathbb{R}}$, where $\varphi: x \mapsto \bar{x}^{\sim}$ is an involutive automorphism on $\mathcal{M}\left(H_{3}(\mathbb{A})\right)_{\mathbb{R}}$. Then

$$
h_{\bar{x}^{\sim}}=h_{\varphi(x)}: z \mapsto(z \bar{x}) \varphi(x)=\left(z \varphi\left(x^{\sim}\right)\right) \varphi(x)=\varphi\left(\left(\varphi(z) x^{\sim}\right) x\right)=\varphi\left(h_{x}(\varphi(z))\right)
$$

for all $x \in \mathcal{M}\left(H_{3}\left(\mathbb{A}^{\mathbb{C}}\right)\right)_{\mathbb{R}}$. Therefore $h_{\bar{x}^{\sim}}=\varphi h_{x} \varphi=\varphi h_{x} \varphi^{-1}$, so $h_{x}$ and $h_{\bar{x}^{\sim}}$ are similar and hence they have the same trace.

Finally, by (3.18), (3.15), (3.14), (3.9) and the last line follows

$$
\gamma_{\phi}(x, x)=2\left(3+\operatorname{dim}_{\mathbb{C}} H_{3}\left(\mathbb{A}^{\mathbb{C}}\right)\right)\left[\left\|\xi_{1}\right\|^{2}+\left\|\xi_{2}\right\|^{2}+T\left(x_{1}, \overline{x_{1}} \wedge\right)+T\left(x_{2}, \overline{x_{2}} \wedge\right)\right]
$$

for all $x=\left(\begin{array}{ll}\xi_{1} & x_{1} \\ x_{2} & \xi_{2}\end{array}\right) \in \mathcal{M}\left(H_{3}\left(\mathbb{A}^{\mathbb{C}}\right)\right)_{\mathbb{R}}$. Then, by $(3.19)$ and Lemma $2, \gamma_{\phi}(x, x)$ is positive definite for all $x \in \mathcal{M}\left(H_{3}\left(\mathbb{A}^{\mathbb{C}}\right)\right)_{\mathbb{R}}$.

We prove now simplicity.

Since the KTS's $\phi(x, y, z)=B_{\mathcal{M}\left(H_{3}\left(\mathbb{A}^{\mathbb{C}}\right)\right)_{\mathbb{R}}}\left(x, \bar{y}^{\sim}, z\right), x, y, z \in \mathcal{M}\left(H_{3}\left(\mathbb{A}^{\mathbb{C}}\right)\right)_{\mathbb{R}}$, are compact then they are simple if and only if the corresponding Kantor algebras $\mathcal{L}(\phi(x, y, z))$ are simple, by [5] Theorem 3.7. Moreover, since $B_{\mathcal{M}\left(H_{3}\left(\mathbb{A}^{\mathbb{C}}\right)\right)_{\mathbb{R}}}(x, y, z), x, y, z \in \mathcal{M}\left(H_{3}\left(\mathbb{A}^{\mathbb{C}}\right)\right)_{\mathbb{R}}$, are KTS's satisfying the condition (A) then the algebras $\mathcal{L}(\phi(x, y, z))$ and $\mathcal{L}\left(B_{\mathcal{M}\left(H_{3}\left(\mathbb{A}^{\mathbb{C}}\right)\right)_{\mathbb{R}}}(x, y, z)\right)$ are isomorphic as

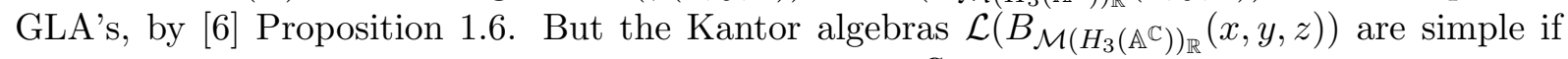
and only if the structurable algebras $\left(A,{ }^{-}\right)=\mathcal{M}\left(H_{3}\left(\mathbb{A}^{\mathbb{C}}\right)\right)_{\mathbb{R}}$ are simple, by [2] Corollary 6 and [7] Theorem 25. Then the simplicity assertion follows from Lemma 1.

Corollary 1. Let $\left(\phi, \mathcal{M}\left(H_{3}\left(\mathbb{A}^{\mathbb{C}}\right)\right)_{\mathbb{R}}\right)$ be the compact KTS's defined by (3.5), (3.7), where $\mathbb{A}^{\mathbb{C}} \in$ $\left\{\mathbb{R}^{\mathbb{C}}, \mathbb{C}^{\mathbb{C}}, \mathbb{H}^{\mathbb{C}}, \mathbb{O}^{\mathbb{C}}\right\}$. Then the canonical quadratic form has the form

$$
\gamma_{\phi}(x, x)=6\left(2+\operatorname{dim}_{\mathbb{C}} \mathbb{A}^{\mathbb{C}}\right)\left[\left\|\xi_{1}\right\|^{2}+\left\|\xi_{2}\right\|^{2}+T\left(x_{1},{\overline{x_{1}}}^{\wedge}\right)+T\left(x_{2},{\overline{x_{2}}}^{\wedge}\right)\right]
$$

for all $x=\left(\begin{array}{ll}\xi_{1} & x_{1} \\ x_{2} & \xi_{2}\end{array}\right) \in \mathcal{M}\left(H_{3}\left(\mathbb{A}^{\mathbb{C}}\right)\right)_{\mathbb{R}}$, where the trace form $T$ is defined by (2.11). 
Proof. The assertion follows from (3.19) since clearly $\operatorname{dim}_{\mathbb{C}}\left(H_{3}\left(\mathbb{A}^{\mathbb{C}}\right)\right)=3\left(1+\operatorname{dim}_{\mathbb{C}} \mathbb{A}^{\mathbb{C}}\right)$.

Remark. By similarity to $[22] \S 2$, define triple systems $\left(\phi^{\prime}, \mathcal{M}\left(H_{3}\left(\mathbb{A}^{\mathbb{C}}\right)\right)_{\mathbb{R}}\right)$ by

$$
\phi^{\prime}(x, y, z)=x\left(y^{\sim} z\right)+z\left(y^{\sim} x\right)-\bar{y}^{\sim}(\bar{x} z), x, y, z \in \mathcal{M}\left(H_{3}\left(\mathbb{A}^{\mathbb{C}}\right)\right)_{\mathbb{R}}
$$

where $\sim$ is the involution on $\mathcal{M}\left(H_{3}\left(\mathbb{A}^{\mathbb{C}}\right)\right)_{\mathbb{R}}$ defined by formula (3.5). Then the triple systems $\left(\phi^{\prime}, \mathcal{M}\left(H_{3}\left(\mathbb{A}^{\mathbb{C}}\right)\right)_{\mathbb{R}}\right)$ are simple compact KTS's, since it can be easily proved that $\left(\phi, \mathcal{M}\left(H_{3}\left(\mathbb{A}^{\mathbb{C}}\right)\right)_{\mathbb{R}}\right)$ and $\left(\phi^{\prime}, \mathcal{M}\left(H_{3}\left(\mathbb{A}^{\mathbb{C}}\right)\right)_{\mathbb{R}}\right)$ are isomorphic under the map $x \mapsto \bar{x}$.

We give the classification theorem. Let Lie algebras be denoted as in [16].

Let $\mathbb{A}^{\mathbb{C}}$ denote any of the complex composition algebras $\mathbb{R}^{\mathbb{C}}, \mathbb{C}^{\mathbb{C}}, \mathbb{H}^{\mathbb{C}}, \mathbb{O}^{\mathbb{C}}$. Let $\mathcal{M}\left(H_{3}\left(\mathbb{A}^{\mathbb{C}}\right)\right)$ be the $2 \times 2$-matrix algebra determined by the Jordan algebra $H_{3}\left(\mathbb{A}^{\mathbb{C}}\right)$ defined by $(2.7)$ with the involutions ${ }^{-}$and $\sim$ defined by (3.5).

Theorem 2. All compact realifications of exceptional simple KTS's defined on the $2 \times 2$ matrix algebra determined by the Jordan algebra $H_{3}\left(\mathbb{A}^{\mathbb{C}}\right)$ are the $\operatorname{KTS}$ 's $\left(\phi, \mathcal{M}\left(H_{3}\left(\mathbb{A}^{\mathbb{C}}\right)\right)_{\mathbb{R}}\right)$, $\mathbb{A}^{\mathbb{C}} \in\left\{\mathbb{R}^{\mathbb{C}}, \mathbb{C}^{\mathbb{C}}, \mathbb{H}^{\mathbb{C}}, \mathbb{O}^{\mathbb{C}}\right\}$, defined by (3.7) and the corresponding Kantor algebras are the following realifications of complex simple Lie algebras $\mathcal{L}\left(\phi, \mathcal{M}\left(H_{3}\left(\mathbb{R}^{\mathbb{C}}\right)\right)_{\mathbb{R}}\right)=F_{4}^{\mathbb{C}}, \mathcal{L}\left(\phi, \mathcal{M}\left(H_{3}\left(\mathbb{C}^{\mathbb{C}}\right)\right)_{\mathbb{R}}\right)=$ $E_{6 \mathbb{R}}^{\mathbb{C}}, \mathcal{L}\left(\phi, \mathcal{M}\left(H_{3}\left(\mathbb{H}^{\mathbb{C}}\right)\right)_{\mathbb{R}}\right)=E_{7 \mathbb{R}}^{\mathbb{C}}, \mathcal{L}\left(\phi, \mathcal{M}\left(H_{3}\left(\mathbb{O}^{\mathbb{C}}\right)\right)_{\mathbb{R}}\right)=E_{8}^{\mathbb{C}}$

Proof. By [15] (Theorem 3.14 and $\S 4.1$ ), in order to classify all compact simple KTS's we have to find one such model for each 5 -grading of each real simple Lie algebra. Moreover, by [16] (Theorem 3.3, Table I), all 5-gradings $\oplus_{l=-2}^{2} K_{l}$ of realifications of complex exceptional simple Lie algebras are such that $\left(\operatorname{dim}_{\mathbb{C}} K_{-1}, \operatorname{dim}_{\mathbb{C}} K_{-2}\right) \in\{(20,5),(20,1),(16,8),(32,10),(32,1),(35,7),(56,1)$, $(64,14),(14,1),(8,7),(4,1)\}$.

Let now $\left(\phi, \mathcal{M}\left(H_{3}\left(\mathbb{A}^{\mathbb{C}}\right)\right)_{\mathbb{R}}\right)$ be the simple compact KTS's defined by (3.7). By the proof

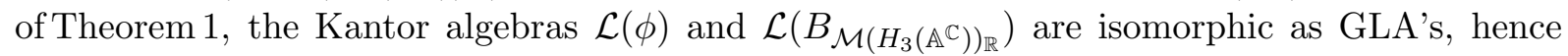
isomorphic to Allison's 5-GLA $\mathcal{K}\left(\mathcal{M}\left(H_{3}\left(\mathbb{A}^{\mathbb{C}}\right)\right)_{\mathbb{R}}\right)$, by [7] Theorem 2.5. Then the assertions follow from (2.6) and [16] (Table I) since it can be easily seen that the only possible $\mathcal{K}\left(\mathcal{M}\left(H_{3}\left(\mathbb{A}^{\mathbb{C}}\right)\right)_{\mathbb{R}}\right)=$ $\oplus_{l=-2}^{2} K_{l}$ are those for which $\left(\operatorname{dim}_{\mathbb{C}} K_{-1}, \operatorname{dim}_{\mathbb{C}} K_{-2}\right) \in\{(14,1),(20,1),(32,1),(56,1)\}$.

\subsection{The exceptional simple Lie algebras $G_{2 \mathbb{R}}^{\mathbb{C}}$ and $G_{2}$}

We give now a close related structure to the one of the previous chapter which leads to models of compact KTS's such that the corresponding Kantor algebra is the real exceptional simple Lie algebra $G_{2 \mathbb{R}}^{\mathbb{C}}$ and moreover the real split $G_{2}$. The approach is closer related to the models of compact KTS's defined in [20] and the presentation of [14], by defining the KTS's on a structurable algebra of skew-dimension one (over $\mathbb{R}$ or $\mathbb{C}$ ), i.e. KTS's defined on a $2 \times 2$-matrix algebra, than the presentation of (complex) KTS's defined on symmetric tensors of [18].

From now on let $\mathbf{F} \in\left\{\mathbb{R}, \mathbb{R}^{\mathbb{C}}=\mathbb{C}\right\}$ and let $\mathcal{M}(\mathbf{F})$ be the algebra with multiplication and standard involution - defined by formula (2.7) [19] (\$4). The algebra $\mathcal{M}(\mathbf{F})$ is called (in the terminology of [2] (§8) the $2 \times 2$-matrix algebra constructed from an admissible non-degenerate cubic form $N$ (with basepoint 1 and scalar 1 ), for short here, the $2 \times 2$-matrix algebra determined by $\mathbf{F}$ (where $N(x)=x^{3}, x \in \mathbf{F}$ ).

Remark. As a direct consequence of the embedding $\mathbf{F} \rightarrow H_{3}(\mathbf{F}), x \mapsto x I_{3}$, where $I_{3}$ is the unit matrix of order 3 , follows $N(x)=x^{3}, \operatorname{Tr}(x)=3 x$, hence

$$
T(x, y)=3 x y \quad \text { and } \quad x \times y=x y, \quad \text { for all } \quad x, y \in \mathbf{F}
$$

by the formulas (2.11). 
Lemma $3([3])$. Let $\left(A,{ }^{-}\right):=\mathcal{M}(\mathbf{F})$ be the $2 \times 2$-matrix algebra determined by $\mathbf{F} \in\{\mathbb{R}, \mathbb{C}\}$, where $\left(^{-}\right)$is the standard involution on $A$. Then, over $\mathbb{R}(\mathbb{C})$, the algebra $\left(A,^{-}\right)$is simple structurable of skew-dimension 1 , if $\mathbf{F}=\mathbb{R}(\mathbb{C})$.

Proof. The assertions follow from [2] (§7, Theorem 11) and [3] (§1, Proposition 1.10).

We define now models of compact simple Kantor triple systems.

Let $\left(A,{ }^{-}\right)=\mathcal{M}(\mathbf{F})$ be the $2 \times 2$-matrix algebra determined by $\mathbf{F} \in\{\mathbb{R}, \mathbb{C}\}$, where ${ }^{-}$is the standard involution on $A$. We define a second involution $\sim$ on $\mathcal{M}(\mathbf{F})$ by

$$
\left(\begin{array}{ll}
\xi_{1} & x_{1} \\
x_{2} & \xi_{2}
\end{array}\right)^{\sim}:=\left(\begin{array}{cc}
\overline{\xi_{1}} & ; \overline{x_{2}} \\
\overline{x_{1}} & \overline{\xi_{2}}
\end{array}\right)
$$

where $\overline{\xi_{i}}, \overline{x_{i}}$ is the standard conjugate of $\xi_{i}, x_{i} \in \mathbf{F}, i=1,2$.

Remark. Clearly, ${ }^{-}$is the identity map in the right hand side of formula (3.21) if $\mathbf{F}=\mathbb{R}$. Moreover, the definition (3.21) is consistent with (3.5), if $\mathbf{F}=\mathbb{C}$, as well as with the definition (2.16) of $[20](\S 2.2)$, if $\mathbf{F}=\mathbb{R}$.

Then the following involutive automorphism is defined on $\mathcal{M}(\mathbf{F})$

$$
\overline{\left(\begin{array}{ll}
\xi_{1} & x_{1} \\
x_{2} & \xi_{2}
\end{array}\right)} \sim\left(\begin{array}{ll}
\overline{\xi_{2}} & \overline{x_{2}} \\
\overline{x_{1}} & \overline{\xi_{1}}
\end{array}\right)
$$

where $\overline{\xi_{i}}, \overline{x_{i}}$ are the standard conjugates of $\xi_{i}, x_{i} \in \mathbf{F}, i=1,2$.

Remark. As above, ${ }^{-}$is the identity map in the right hand side of formula (3.22) if $\mathbf{F}=\mathbb{R}$.

Let $\mathcal{M}(\mathbb{C})_{\mathbb{R}}$ denote the realification of the algebra $\mathcal{M}(\mathbb{C})$. Then we have

Proposition 4. The triple systems $\left(\phi, \mathcal{M}(\mathbb{C})_{\mathbb{R}}\right)$ and $(\phi, \mathcal{M}(\mathbb{R}))$ defined by (3.7), (3.21) are KTS's satisfying the condition (A).

Proof. For the case $\left(\phi, \mathcal{M}(\mathbb{C})_{\mathbb{R}}\right)$ the proof is identical to the proof of Proposition 3, by replacing in the proof of Proposition 3 the algebra $\mathcal{M}\left(H_{3}\left(\mathbb{A}^{\mathbb{C}}\right)\right)_{\mathbb{R}}$ with $\mathcal{M}(\mathbb{C})_{\mathbb{R}}$ and Lemma 1 with Lemma 3 , respectively. Further, for the case $(\phi, \mathcal{M}(\mathbb{R}))$ the proof is identical to the proof of [20] Proposition 2.4 , by replacing in the proof of $[20]$ Proposition 2.4 the algebra $\mathcal{M}\left(H_{3}(\mathbb{A})\right)$ with $\mathcal{M}(\mathbb{R})$ and $[20]$ Lemma 1.2 with Lemma 3, respectively.

We give now the analog of Theorem 1 and [20] Theorem 2.1.

Theorem 3. The KTS's $\left(\phi, \mathcal{M}(\mathbb{C})_{\mathbb{R}}\right)$ and $(\phi, \mathcal{M}(\mathbb{R}))$ defined by (3.7),(3.21) are compact, simple.

Proof. We prove first compactness. We must show that the canonical (trace) form $\gamma_{\phi}$ defined by $(2.4)$ for the KTS's $\left(\phi, \mathcal{M}(\mathbb{C})_{\mathbb{R}}\right)$ and $(\phi, \mathcal{M}(\mathbb{R}))$, respectively, is positive definite. Since the canonical form is symmetric we consider the corresponding quadratic form (3.8). Then, by (3.19) and (3.20),

$$
\gamma_{\phi}(x, x)=2\left(3+\operatorname{dim}_{\mathbb{C}} \mathbb{C}\right)\left(\left\|\xi_{1}\right\|^{2}+\left\|\xi_{2}\right\|^{2}+3\left\|x_{1}\right\|^{2}+3\left\|x_{2}\right\|^{2}\right)
$$

for all $x=\left(\begin{array}{ll}\xi_{1} & x_{1} \\ x_{2} & \xi_{2}\end{array}\right) \in \mathcal{M}(\mathbb{C})_{\mathbb{R}}$, where $\|c\|$ denotes the norm of $c \in \mathbb{C}$. Then, by $(3.23), \gamma_{\phi}(x, x)=$ $8\left(\left\|\xi_{1}\right\|^{2}+\left\|\xi_{2}\right\|^{2}+3\left\|x_{1}\right\|^{2}+3\left\|x_{2}\right\|^{2}\right)$ hence $\gamma_{\phi}(x, x)$ is positive definite for all $x \in \mathcal{M}(\mathbb{C})_{\mathbb{R}}$. Analogously, by [20] (2.29) and (3.20),

$$
\gamma_{\phi}(x, x)=\left(3+\operatorname{dim}_{\mathbb{R}} \mathbb{R}\right)\left(\xi_{1}^{2}+\xi_{2}^{2}+3 x_{1}^{2}+3 x_{2}^{2}\right)
$$


for all $x=\left(\begin{array}{ll}\xi_{1} & x_{1} \\ x_{2} & \xi_{2}\end{array}\right) \in \mathcal{M}(\mathbb{R})$. Then, by $(3.24), \gamma_{\phi}(x, x)=4\left(\xi_{1}^{2}+\xi_{2}^{2}+3 x_{1}^{2}+3 x_{2}^{2}\right)$ hence $\gamma_{\phi}(x, x)$ is positive definite for all $x \in \mathcal{M}(\mathbb{R})$.

We prove now simplicity.

For the case $\left(\phi, \mathcal{M}(\mathbb{C})_{\mathbb{R}}\right)$ the proof is identical to the proof of the simplicity assertion of Theorem 1 , by replacing in the proof of Theorem 1 the algebra $\mathcal{M}\left(H_{3}\left(\mathbb{A}^{\mathbb{C}}\right)\right)_{\mathbb{R}}$ with $\mathcal{M}(\mathbb{C})_{\mathbb{R}}$ and Lemma 1 with Lemma 3 , respectively. Further, for the case $(\phi, \mathcal{M}(\mathbb{R}))$ the proof is identical to the proof of the simplicity assertion of [20] Theorem 2.1, by replacing in the proof of [20] Theorem 2.1 the algebra $\mathcal{M}\left(H_{3}(\mathbb{A})\right)$ with $\mathcal{M}(\mathbb{R})$ and $[20]$ Lemma 1.2 with Lemma 3, respectively.

Remark. By similarity to [22] $\S 2$, define triple systems $\left(\phi^{\prime}, \mathcal{M}\left(H_{3}\left(\mathbb{A}^{\mathbb{C}}\right)\right)_{\mathbb{R}}\right)$ by

$$
\phi^{\prime}(x, y, z)=x\left(y^{\sim} z\right)+z\left(y^{\sim} x\right)-\bar{y}^{\sim}(\bar{x} z), x, y, z \in \mathcal{M}(\mathbb{C})_{\mathbb{R}}
$$

where $\sim$ is the involution on $\mathcal{M}(\mathbb{C})_{\mathbb{R}}$ defined by $(3.21)$. Then the triple systems $\left(\phi^{\prime}, \mathcal{M}(\mathbb{C})_{\mathbb{R}}\right)$ are simple compact KTS's, since it can be easily checked that $\left(\phi, \mathcal{M}(\mathbb{C})_{\mathbb{R}}\right)$ and $\left(\phi^{\prime}, \mathcal{M}(\mathbb{C})_{\mathbb{R}}\right)$ are isomorphic under the map $x \mapsto \bar{x}$.

Analogously, the triple systems $\left(\phi^{\prime}, \mathcal{M}(\mathbb{R})\right)$ are simple compact KTS's.

Proposition 5. Let $\left(\phi, \mathcal{M}(\mathbb{C})_{\mathbb{R}}\right)$ and $(\phi, \mathcal{M}(\mathbb{R}))$ be the KTS's defined by (3.7), (3.21). Then the corresponding Kantor algebras are the exceptional simple Lie algebras $\mathcal{L}\left(\phi, \mathcal{M}(\mathbb{C})_{\mathbb{R}}\right)=G_{2}^{\mathbb{C}}$ and $\mathcal{L}(\phi, \mathcal{M}(\mathbb{R}))=G_{2}$.

Proof. The proof is based on dimensional reasons. Consider first the simple compact KTS $\left(\phi, \mathcal{M}(\mathbb{C})_{\mathbb{R}}\right)$. By [6] Proposition 1.6, the Kantor algebras $\mathcal{L}(\phi)$ and $\mathcal{L}\left(B_{\mathcal{M}(\mathbb{C})_{\mathbb{R}}}\right)$ are isomorphic as GLA's, hence isomorphic to Allison's 5-GLA $\mathcal{K}\left(\mathcal{M}(\mathbb{C})_{\mathbb{R}}\right)$, by [7] Theorem 2.5. Then the assertion follow from (2.6) and [16] (Table I) since it can be easily seen that the only possible $\mathcal{K}\left(\mathcal{M}(\mathbb{C})_{\mathbb{R}}\right)=\oplus_{l=-2}^{2} K_{l}$ with $\left(\operatorname{dim}_{\mathbb{C}} K_{-1}, \operatorname{dim}_{\mathbb{C}} K_{-2}\right)=(4,1)$ is $G_{2 \mathbb{R}}^{\mathbb{C}}$.

Analogously, consider the simple compact $\operatorname{KTS}(\phi, \mathcal{M}(\mathbb{R}))$. By [6] Proposition 1.6, the Kantor algebras $\mathcal{L}(\phi)$ and $\mathcal{L}\left(B_{\mathcal{M}(\mathbb{R})}\right)$ are isomorphic as GLA's, hence isomorphic to Allison's 5-GLA $\mathcal{K}(\mathcal{M}(\mathbb{R}))$, by [7] Theorem 2.5. Then the assertion follow from (2.6) and [16] (Table I) since it can be easily seen that the only possible $\mathcal{K}(\mathcal{M}(\mathbb{R}))=\oplus_{l=-2}^{2} K_{l}$ with $\left(\operatorname{dim}_{\mathbb{R}} K_{-1}\right.$, $\left.\operatorname{dim}_{\mathbb{R}} K_{-2}\right)=(4,1)$ is $G_{2}$.

Remark. The identity $\mathcal{L}\left(B_{\mathcal{M}(\mathbb{R})}\right)=G_{2}$ follows also from [2] ( $\S 8$, p. 1871).

\section{Acknowledgements}

The author is grateful to Professor A. Elduque and the Department of Mathematics, University of Zaragoza, Spain, for a research visit during June 2005 and acknowledges partial support for this research from the Spanish Ministerio de Educatión y Ciencia (MTM 2004-081159-C04-02).

\section{References}

[1] B. N. Allison. A class of nonassociative algebras with involution containing the class of Jordan algebras. Math. Ann. 237 (1978), 133-156.

[2] B. N. Allison. Models of isotropic simple Lie algebras. Comm. Algebra 7 (1979), 1835-1875.

[3] B. N. Allison. Simple structurable algebras of skew-dimension one. Comm. Algebra 18 (1990), 1245-1279.

[4] B. N. Allison and J. R. Faulkner. Elementary groups and invertibility for Kantor pairs. Comm. Algebra 27 (1999), 519-556. 
[5] H. Asano and S. Kaneyuki. On compact generalized Jordan triple systems of the second kind. Tokyo J. Math. 11 (1) (1988), 105-118 and Correction. Tokyo J. Math. 15 (1992), 483-484.

[6] H. Asano. Classification of non-compact real simple generalized Jordan triple systems of the second kind. Hiroshima Math. J. 21 (1991), 463-489.

[7] H. Asano and S. Kaneyuki. Another construction of Allison's graded Lie algebras obtained from structurable algebras. Yokohama Math. J. 46 (1998), 87-96.

[8] H. Braun and M. Koecher. Jordan-Algebren. Springer-Verlag, Berlin, 1966.

[9] H. Freudenthal. Beziehungen der $E_{7}$ und $E_{8}$ zur Oktavenebene. I. Indag. Math. 16 (1954), 218-230.

[10] S. Gomyo. A construction of exceptional simple graded Lie algebras of the second kind. Proc. Japan Acad. Ser. A Math. Sci. 71 (1995), 13-16.

[11] N. Jacobson. Lie algebras. Interscience Publishers, New York, 1962.

[12] N. Jacobson. Structure and representations of Jordan algebras. Amer. Math. Soc. Colloq. Publ., vol. 39, Amer. Math. Soc., Providence, R.I., 1968.

[13] N. Kamiya. A structure theory of Freudenthal-Kantor triple systems. II. Comment. Math. Univ. St. Paul 38 (1989), 41-60.

[14] N. Kamiya. On a realization of exceptional simple graded Lie algebras of the second kind and Freudenthal-Kantor triple systems. Bull. Polish Acad. Sci. Math. 46 (1998), 55-65.

[15] S. Kaneyuki and H. Asano. Graded Lie algebras and generalized Jordan triple systems. Nagoya Math. J. 112 (1988), 81-115.

[16] S. Kaneyuki. On the subalgebras $\mathfrak{g}_{0}$ and $\mathfrak{g}_{\mathrm{ev}}$ of semisimple graded Lie algebras. J. Math. Soc. Japan 45 (1993), 1-19.

[17] I. L. Kantor. Some generalizations of Jordan algebras. Trudy Sem. Vect. Tens. Anal. 16 (1972), 407-499.

[18] I. L. Kantor. Models of exceptional Lie algebras. Soviet Math. Dokl. Vol. 14 (1973), 254-258.

[19] K. McCrimmon. The Freudenthal-Springer-Tits constructions of exceptional Jordan algebras. Trans. Amer. Math. Soc. 139 (1969), 495-510.

[20] D. Mondoc. Models of compact simple Kantor triple systems defined on a class of structurable algebras of skew-dimension one. Comm. Algebra 34 (2006), 3801-3815.

[21] D. Mondoc. Compact exceptional simple Kantor triple systems defined on tensor products of composition algebras. Comm. Algebra, 2007 (to be published).

[22] D. Mondoc. Compact realifications of exceptional simple Kantor triple systems defined on tensor products of composition algebras. J. Algebra 307 (2007), 917-929.

[23] R. D. Schafer. An introduction to nonassociative algebras. Academic Press, New York, 1966.

[24] K. Yamaguti and A. Ono. On representations of Freudenthal-Kantor triple systems $\mathfrak{U}(\epsilon, \delta)$. Bull. Fac. School Ed. Hiroshima Univ. II (7) (1984), 43-51. 